\title{
Volume of cerebrospinal fluid drainage as a predictor for pretreatment aneurysmal rebleeding
}

\author{
*Jasper H. van Lieshout, MD, ${ }^{1}$ Ina Pumplün, ${ }^{1}$ Igor Fischer, PhD,, Marcel A. Kamp, MD,1 \\ Jan F. Cornelius, MD, PhD, ${ }^{1}$ Hans J. Steiger, MD, PhD, ${ }^{1}$ Hieronymus D. Boogaarts, MD, PhD, ${ }^{2}$ \\ Athanasios K. Petridis, MD, PhD, ${ }^{1}$ and Kerim Beseoglu, MD, PhD' \\ 'Department of Neurosurgery, Medical Faculty, Heinrich-Heine University Düsseldorf, Germany; and 2Department of \\ Neurosurgery, Radboud University Medical Center, Nijmegen, The Netherlands
}

\begin{abstract}
OBJECTIVE Initiation of external CSF drainage has been associated with a significant increase in rebleeding probability after aneurysmal subarachnoid hemorrhage (aSAH). However, the implications for acute management are uncertain. The purpose of this study was to evaluate the role of the amount of drained CSF on aneurysmal rebleeding.
\end{abstract}

METHODS Consecutive patients with aSAH were analyzed retrospectively. Radiologically confirmed cases of aneurysmal in-hospital rebleeding were identified and predictor variables for rebleeding were retrieved from hospital records. Clinical predictors were identified through multivariate analysis, and logistic regression analysis was performed to ascertain the cutoff value for the rebleeding probability.

RESULTS The study included 194 patients. Eighteen cases (9.3\%) of in-hospital rebleeding could be identified. Using multivariate analysis, in-hospital rebleeding was significantly associated with initiation of CSF drainage $(p=0.001)$ and CSF drainage volume (63 ml [interquartile range (IQR) 55-69 ml] vs $25 \mathrm{ml}$ [IQR 10-35 ml], $p<0.001$ ). Logistic regression showed that $58 \mathrm{ml}$ of CSF drainage within 6 hours results in a 50\% rebleeding probability. The relative risk (RR) for rebleeding after drainage of more than $60 \mathrm{ml}$ in 6 hours was 5.4 times greater compared with patients with less CSF drainage (RR $5.403,95 \% \mathrm{Cl} 2.481-11.767 ; p<0.001$, number needed to harm $=1.687$ ).

CONCLUSIONS Volume of CSF drainage was highly correlated with the probability of in-hospital aneurysmal rebleeding. These findings suggest that the rebleeding probability can be affected in acute management should the placement of an external ventricular catheter be necessary. This finding necessitates meticulous control of the amount of drained CSF and the development of a definitive treatment protocol for this group of patients.

https://thejns.org/doi/abs/10.3171/2017.2.JNS162748

KEY WORDS subarachnoid hemorrhage; intracranial aneurysm; recurrence; risk factor; cerebrospinal fluid; external ventricular drainage; vascular disorders

$\mathrm{T}$ HE incidence of aneurysmal subarachnoid hemorrhage (aSAH) ranges from 2 to 23 cases per 100,000 and is associated with significant morbidity and mortality. ${ }^{9,10} \mathrm{Of}$ all patients with aSAH, an estimated 5.8\%-22\% will suffer from aneurysmal rebleeding. 6,7 , 12,14,22 These events usually occur within the first 24 hours of the primary bleed and have an associated mortality rate of $50 \%-60 \% .{ }^{12,14}$ Because rebleeding significantly impairs prognosis, its prevention is one of the primary goals of acute management. ${ }^{6,14}$

However, concurrent challenges such as acute hydro- cephalus or the need for invasive neuromonitoring may demand the use of an external ventricular drain (EVD), which leads to changes in transmural pressure across the aneurysm wall. Changes in transmural pressure have long been regarded as a possible cause for rebleeding, and studies have shown that the initiation of external CSF drainage is associated with a significant increase in rebleeding probability. $8,15,22$

In clinical practice, it remains unclear whether this intervention complicates acute management and what consequences for patients with aSAH should be drawn. In this

ABBREVIATIONS aSAH = aneurysmal subarachnoid hemorrhage; $\mathrm{Cl}=$ confidence interval; $E V D=$ external ventricular drain; ICP = intracranial pressure; $I Q R=$ interquartile range; $\mathrm{NNH}$ = number needed to harm; RR = relative risk; SBP = systolic blood pressure; WFNS = World Federation of Neurosurgical Societies.

SUBMITTED October 31, 2016. ACCEPTED February 2, 2017.

INCLUDE WHEN CITING Published online August 11, 2017; DOI: 10.3171/2017.2.JNS162748.

* Drs. Petridis and Beseoglu contributed equally to this work. 
context, we aimed to identify risk factors for in-hospital rebleeding before aneurysm occlusion and focus on the effect of CSF drainage on the incidence of aneurysmal rebleeding.

\section{Methods}

\section{Study Population}

We conducted a retrospective study of 194 consecutive patients with radiologically confirmed aSAH admitted to the Heinrich-Heine University Medical Centre between May 2012 and January 2016. The ethics committee of Heinrich-Heine University Medical Centre approved this study.

\section{Treatment Protocol}

A standardized treatment protocol is applied to all patients admitted to our center with aSAH as described elsewhere. ${ }^{4,21}$ All patients undergo immediate CT angiography and perfusion CT upon admission. Patients are monitored on our neurointensive care unit under a minimal touch regimen until aneurysm closure. The systolic blood pressure (SBP) is lowered so as not to exceed $140 \mathrm{~mm} \mathrm{Hg}$. Antifibrinolytic agents are only applied when definite aneurysm closure cannot be achieved within 36 hours.

\section{EVD Management and CSF Drainage}

In patients with a Glasgow Coma Scale score below 13 , invasive neuromonitoring with insertion of an intraventricular catheter is initiated. All patients adhered to a standardized drainage protocol. The intracranial pressure (ICP) threshold is set at $18 \mathrm{~mm} \mathrm{Hg}$. ICP is monitored continuously and CSF is drained in case of sustained elevation of ICP above the set limit. When the ICP exceeded 18 $\mathrm{mm} \mathrm{Hg}$ for a period longer than 15 minutes, the EVD was opened at a hydraulic pressure level of $18 \mathrm{~mm} \mathrm{Hg}$. Drainage of CSF was halted immediately when ICP dropped to $18 \mathrm{~mm} \mathrm{Hg}$ and none of the patients were treated under a continuous hydraulic drainage paradigm. The drained volume of CSF is documented hourly.

\section{Data Collection and Definitions}

For all 194 included patients epidemiological, clini- cal, and radiological data were collected. From this study population the number of rebleeding events was identified. Aneurysmal rebleeding was defined as CT-confirmed episodes of in-hospital rebleeding in which imaging was prompted by any neurological deterioration, or otherwise clinical suspicious events in comatose patients, such as bradycardia, sudden rise in blood pressure, or the appearance of fresh blood through ventricular drainage. ${ }^{2}$ The amount and intensity of blood was compared with that on the preceding CT. Rebleeding before hospitalization was registered; events after aneurysmal obliteration were not included. For further analysis, only patients with CSF drainage initiated at least 6 hours before aneurysm closure were included to allow for uniform analysis of the retrospective data (Fig. 1). One hundred seventeen patients were excluded from secondary analysis because CSF drainage had been started after aneurysm closure $(n=45)$, or no CSF drainage was initiated $(n=72)$. Another 12 patients were excluded because of incomplete data or variable duration of CSF drainage. Therefore, 65 patients (21 men [32.3\%], mean age $58.6 \pm 12.6$ years) were included for further analysis (Fig. 1). For the included patients we registered the volume of CSF drainage at 6,12 , and 24 hours before aneurysm closure.

\section{Statistical Analysis}

Categorical data are presented as counts and percentages and continuous variables as means with standard deviations (SDs) or medians with interquartile ranges (IQRs), depending on the normality of data.

We performed univariate analysis of all clinically important covariates (Table 1). For categorical data (Fisher grade, World Federation of Neurosurgical Societies [WFNS] grade) we discriminated between a poor grade and a good grade due to the strong dichotomy: WFNS grade $\leq 3$ and $\geq 4$, Fisher grade $<4$ and $=4$. Variables were tested for an association with rebleeding using the Fisher exact test (grouped data), t-test (for continuous normally distributed variables), or the Mann-Whitney test (for continuous, nonnormally distributed variables). Multivariate analysis was performed to identify factors associated with aneurysmal rebleeding. We considered a 2 -tailed $p$ value of 0.05 statistically significant. Binary logistic regres-

194 Patients with aneurysmal subarachnoid hemorrhage

\begin{tabular}{|l|l|}
117 Excluded \\
45 Patients received EVD after aneurysm closure \\
72 Patients did not receive CSF drainage
\end{tabular}

FIG. 1. Flowchart demonstrating the selection process for the study. 
TABLE 1. Univariate and multivariate factors related to a rebleeding event in 194 patients with aSAH

\begin{tabular}{|c|c|c|c|c|c|}
\hline \multirow[b]{2}{*}{ Factor } & \multicolumn{3}{|c|}{ No. of Patients (\%) } & \multicolumn{2}{|c|}{$p$ Value } \\
\hline & All Patients & Rebleeding & Nonrebleeding & Univariate Analysis & Multivariate Analysis \\
\hline Total & $194(100)$ & $26(13.4)$ & $168(86.6)$ & - & - \\
\hline Females & $116(59.8)$ & $16(61.5)$ & $100(59.5)$ & - & - \\
\hline Males & $78(40.2)$ & $10(38.5)$ & $68(40.5)$ & - & - \\
\hline Mean age \pm SD (yrs) & $55.6 \pm 12.4$ & $60.3 \pm 12.4$ & $54.8 \pm 12.3$ & - & - \\
\hline \multicolumn{6}{|l|}{ WFNS grade on admission } \\
\hline 1 & $61(31.4)$ & $0(0)$ & $61(36.3)$ & - & - \\
\hline 2 & $27(13.9)$ & $1(3.8)$ & $26(15.4)$ & - & - \\
\hline 3 & $16(8.2)$ & $5(19.2)$ & $11(6.5)$ & - & - \\
\hline 4 & $31(16.0)$ & $3(11.5)$ & $28(16.7)$ & $<0.001$ & 0.010 \\
\hline 5 & $59(30.4)$ & $17(65.4)$ & $42(25)$ & - & - \\
\hline Fisher grade on admission & & & & - & - \\
\hline 1 & $10(5.2)$ & $0(0)$ & $10(6)$ & - & - \\
\hline 2 & $7(3.6)$ & $0(0)$ & $7(4.2)$ & - & - \\
\hline 3 & $50(25.8)$ & $1(3.8)$ & $49(29.2)$ & - & - \\
\hline 4 & $127(65.5)$ & $25(96.2)$ & $102(60.7)$ & $<0.001$ & 0.144 \\
\hline Aneurysm size $(\mathrm{mm})^{*}$ & & & & 0.059 & 0.095 \\
\hline 0 to $<5$ & $69(35.6)$ & $4(15.4)$ & $65(38.7)$ & - & - \\
\hline 5 to $<10$ & $99(51)$ & $16(61.5)$ & $83(49.4)$ & - & - \\
\hline 10 to $<15$ & $20(10.3)$ & $5(19.2)$ & $15(8.9)$ & - & - \\
\hline 15 to $<20$ & $2(1)$ & $1(3.8)$ & $1(0.6)$ & - & - \\
\hline$>20$ & $4(2.1)$ & $0(0)$ & $4(2.4)$ & - & - \\
\hline Aneurysm location & & & & 0.344 & - \\
\hline Anterior & $134(69.1)$ & $17(65.4)$ & $117(69.6)$ & - & - \\
\hline Posterior & $60(30.9)$ & $9(34.6)$ & $51(30.4)$ & - & - \\
\hline External CSF drainage before aneurysm closure & & & & $<0.001$ & 0.001 \\
\hline Yes & $77(39.7)$ & $17(94.4)$ & $60(34.1)$ & - & - \\
\hline No & $117(60.3)$ & $1(5.6)$ & $116(65.9)$ & - & - \\
\hline
\end{tabular}

Boldface type indicates statistical significance.

* Missing data: aneurysm size, $n=1(0.5 \%)$.

sion analysis was performed to ascertain the cutoff point from the variable identified through multivariate analysis for the prediction of rebleeding. For the patients with a volume of CSF drainage above the $50 \%$ probability line in the binary logistic regression analysis, we calculated a relative risk with $95 \%$ confidence interval (CI) and number needed to harm (NNH) to cause one rebleeding due to high volume CSF drainage. Correlation (R) between individual variables was calculated using Pearson's productmoment correlation. All statistical analysis was performed using the $\mathrm{R}$ statistical computing package (version 3.2.2, https://r-project.org/).

\section{Results}

\section{Patient Demographics and Rebleeding Incidence}

The characteristics of the study population are listed in Table 1. Pretreatment rebleeding was radiologically confirmed in $26(13.4 \%)$ of 194 patients. These 26 patients had a total of 28 rebleeding events. Ten (5.2\%) of these patients suffered a rebleeding event during transport from the referring hospital to our clinic. Two patients had both an out-of-hospital and in-hospital rebleeding event, resulting in a $9.3 \%(n=18)$ in-hospital rebleeding rate. The time distribution of patients with in-hospital rebleeding is depicted in Fig. 2.

\section{Interventions}

The median time to aneurysm repair in all patients was 15 hours (IQR 6-23 hours) after admission; the median time for EVD placement was 3 hours (IQR 2-5 hours). Of the 77 patients who received CSF drainage before aneurysm closure, 17 (22.1\%) suffered from aneurysmal rebleeding. Two patients experienced aneurysmal rebleeding shortly after initiation of CSF drainage, and no documented amount of drained CSF volume could be retrieved from the charts. There was no difference in duration of CSF drainage between the rebleeding and nonrebleeding group (17 hours [IQR 7.5-21.5 hours] vs 14 hours [IQR 8.5-21 hours], $\mathrm{p}=0.58)$. In total, 65 patients $(33.5 \%)$ underwent CSF drainage over the course of at least 6 hours. Characteristics of these patients are listed in Table 2. Rebleeding occurred in $15(23.0 \%)$ of these patients. In 25 


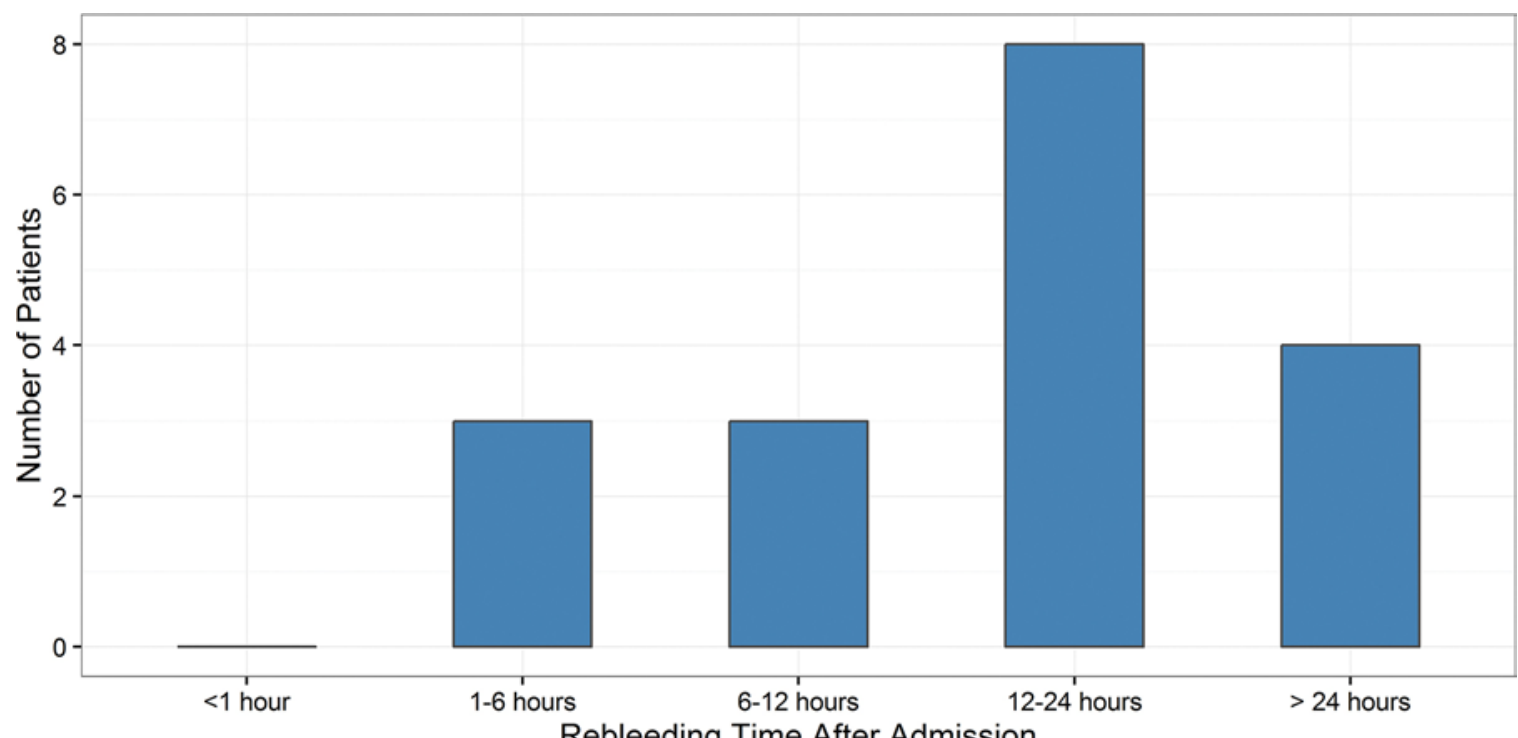

Rebleeding Time After Admission

FIG. 2. Graph of the time interval between admission and in-hospital aneurysmal rebleeding. Figure is available in color online only.

cases (12.9\%), CSF drainage continued over the course of 24 hours and 4 of the 15 rebleeding events occurred after this timeframe. Of these 4 patients, aneurysm closure was delayed because of cardiac or respiratory complications (in 3 patients) or logistic reasons (in 1 patient). None of the patients was treated with antifibrinolytic agents.

\section{Risk Factors for Aneurysmal Rebleeding}

In multivariate regression analysis the independent predictors for aneurysmal rebleeding were WFNS grade $\geq 4(p=0.010)$ and the initiation of CSF drainage before aneurysm closure $(p=0.001$; Table 1$)$. Factors related to aneurysmal rebleeding in patients with an EVD before aneurysm closure are presented in Table 2 . The rebleedingpositive group had a significantly higher volume of CSF drainage before aneurysmal rebleeding compared with the rebleeding-negative group (Fig. 3; $63 \mathrm{ml}$ [IQR 55-69 ml] vs $25 \mathrm{ml}$ [IQR 10-35 ml], p < 0.001). In logistic regression analysis, a drained volume of $58 \mathrm{ml}$ within 6 hours was identified as the cutoff value; the lower range of the 95\% CI establishes the 50\% probability line at $49 \mathrm{ml}$ (Fig. 4). At 12 hours after EVD insertion the effect remained statistically significant (cutoff value $122 \mathrm{ml}$, lower 95\% CI $98.5 \mathrm{ml} ; \mathrm{p}<0.001)$. However, the effect was lost after 24 hours as patients were excluded from further analysis due to aneurysm closure $(\mathrm{p}=0.062)$. The relative risk $(\mathrm{RR})$ for rebleeding after drainage of more than $60 \mathrm{ml}$ in 6 hours is 5.4 times greater compared with patients with less CSF drainage (RR 5.403, 95\% CI 2.481-11.767; p < 0.001, NNH 1.687).

Independent predictors for a high volume of CSF drainage were aneurysm diameter $(\mathrm{R}=0.301,95 \% \mathrm{CI} 0.058$ $0.511, \mathrm{p}=0.016)$ and WFNS grade $(\mathrm{R}=0.263,95 \% \mathrm{CI}$ $0.016-0.479, \mathrm{p}=0.038$; Table 3).

TABLE 2. Univariate and multivariate factors related to a rebleeding event in 65 patients with EVD before aneurysm closure

\begin{tabular}{|c|c|c|c|c|}
\hline \multirow[b]{2}{*}{ Factor } & \multicolumn{2}{|c|}{ Rebleeding } & \multicolumn{2}{|c|}{$p$ Value } \\
\hline & Positive & Negative & Univariate Analysis & Multivariate Analysis \\
\hline No. of patients & 15 & 50 & & \\
\hline WFNS grade $\geq 4(\%)$ & $12(80.0)$ & $34(68.0)$ & 0.522 & - \\
\hline Fisher grade $>3(\%)$ & $14(93.3)$ & $41(82.0)$ & 0.431 & - \\
\hline Aneurysm diameter $>10 \mathrm{~mm}(\%)$ & $9(60.0)$ & $8(16.0)$ & 0.02 & 0.081 \\
\hline \multicolumn{5}{|l|}{ Aneurysm location (\%) } \\
\hline Anterior & $10(67.7)$ & $30(60.0)$ & 0.767 & - \\
\hline Posterior & $5(33.3)$ & $20(40.0)$ & & \\
\hline Median amount of CSF drainage in $6 \mathrm{hrs}$ in $\mathrm{ml}(\mathrm{IQR})$ & $63(55-69)$ & $25(10-35)^{*}$ & $<0.001$ & $<0.001$ \\
\hline Median time of EVD insertion after admission in hrs (IQR) & $2(1-2.5)$ & $3(2-6)$ & 0.390 & - \\
\hline Thrombocytopenia (\%) & $5(33.3)$ & $0(0.0)$ & $<0.001$ & 0.993 \\
\hline
\end{tabular}

Boldface type indicates statistical significance.

* Missing data: CSF drainage in 6 hours, $n=2(3 \%)$. 


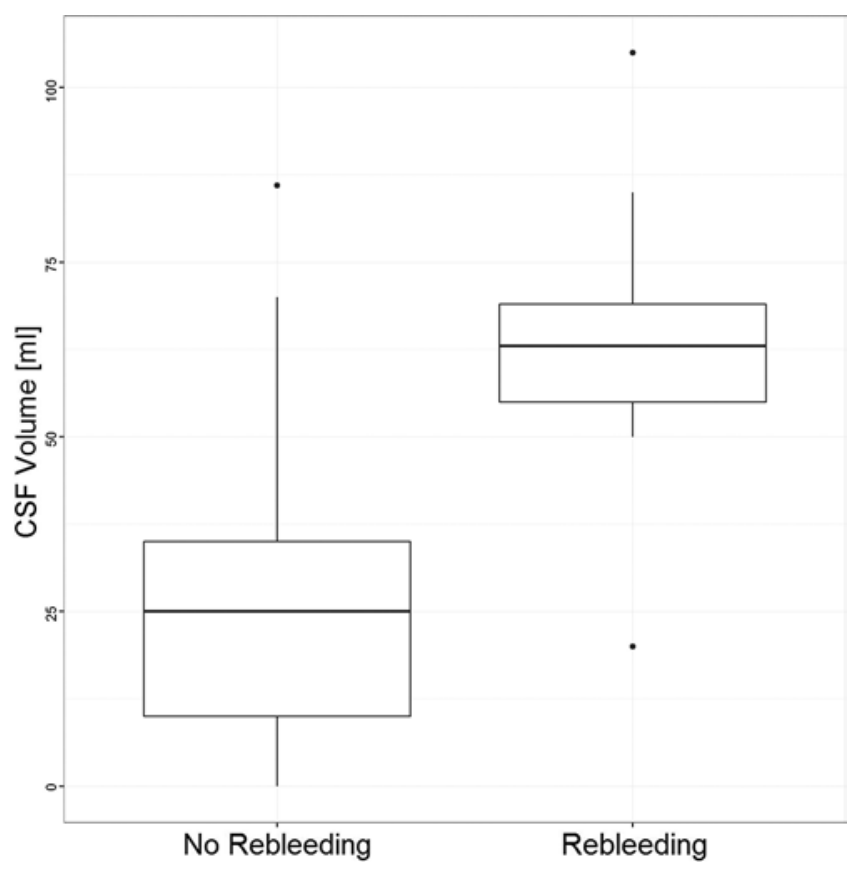

FIG. 3. Box plot visualizing the difference in CSF drainage between the rebleeding and nonrebleeding groups.

We did not find a significant difference in maximal SBP during admission between the rebleeding and the nonrebleeding group $(152 \pm 24 \mathrm{~mm} \mathrm{Hg}$ vs $150 \pm 20 \mathrm{~mm} \mathrm{Hg}, \mathrm{p}=$ $0.737)$, nor was there a difference with the maximum SBP within the 30 minutes before aneurysmal rebleeding (126 $\pm 17 \mathrm{~mm} \mathrm{Hg}$ vs $150 \pm 20 \mathrm{~mm} \mathrm{Hg}$ ). We also did not observe more SBP spikes between the rebleeding and the nonrebleeding groups (above $140 \mathrm{~mm} \mathrm{Hg}, \mathrm{p}=1.000$, or $160 \mathrm{~mm}$ $\mathrm{Hg}, \mathrm{p}=0.740)$. There were no significant differences in international normalized ratio $(1.07 \pm 0.07$ vs $1.04 \pm 0.06$, $\mathrm{p}=0.099)$, partial thromboplastin time $(23.3 \pm 3.6$ vs 25.9
TABLE 3. Independent predictive value for amount of CSF drainage

\begin{tabular}{lcrc}
\hline Independent Predictor & Correlation & $95 \% \mathrm{Cl}$ & $\mathrm{p}$ Value \\
\hline WFNS grade & 0.263 & 0.016 to 0.479 & 0.038 \\
\hline Fisher grade & 0.114 & -0.138 to 0.352 & 0.374 \\
\hline Aneurysm diameter & 0.301 & 0.058 to 0.511 & $\mathbf{0 . 0 1 6}$ \\
\hline Aneurysm location & 0.182 & -0.073 to 0.411 & 0.091 \\
\hline
\end{tabular}

Boldface type indicates statistical significance.

$\pm 11.4, \mathrm{p}=0.388)$, antiplatelet therapy $(\mathrm{p}=0.153)$, or oral anticoagulation $(\mathrm{p}=0.381)$. The absolute thrombocyte count did not differ between the rebleeding $(222 \times 1000 /$ $\mu \mathrm{l} \pm 101)$ and nonrebleeding groups $(226 \times 1000 / \mu \mathrm{l} \pm 56, \mathrm{p}$ $=0.869)$. Five patients fulfilled the criteria for thrombocytopenia (thrombocyte count below $150 \times 1000 / \mu \mathrm{l})$ and all belonged to the rebleeding group $(\mathrm{p}<0.001)$. When the presence of thrombocytopenia was included in the multivariate analysis it lost significance due to the strong effect of CSF drainage ( $\mathrm{p}=0.993$, Table 2$)$.

\section{Discussion}

This study revealed some novel issues about management of aSAH before aneurysm closure. First, initiation of external ventricular CSF drainage is independently associated with pretreatment rebleeding. Second, the amount of CSF volume drained is independently correlated with the probability of rebleeding.

Among other factors, such as clinical condition, hypertension, aneurysm size, and amount of subarachnoid blood, the initiation of CSF drainage is an independent risk factor for a rebleeding event after aSAH., $3,5,7,12-14,19,22$ Moreover, its deleterious effect during aneurysmal rerupture suggested CSF drainage should be avoided during recurrent hemorrhages. ${ }^{23}$

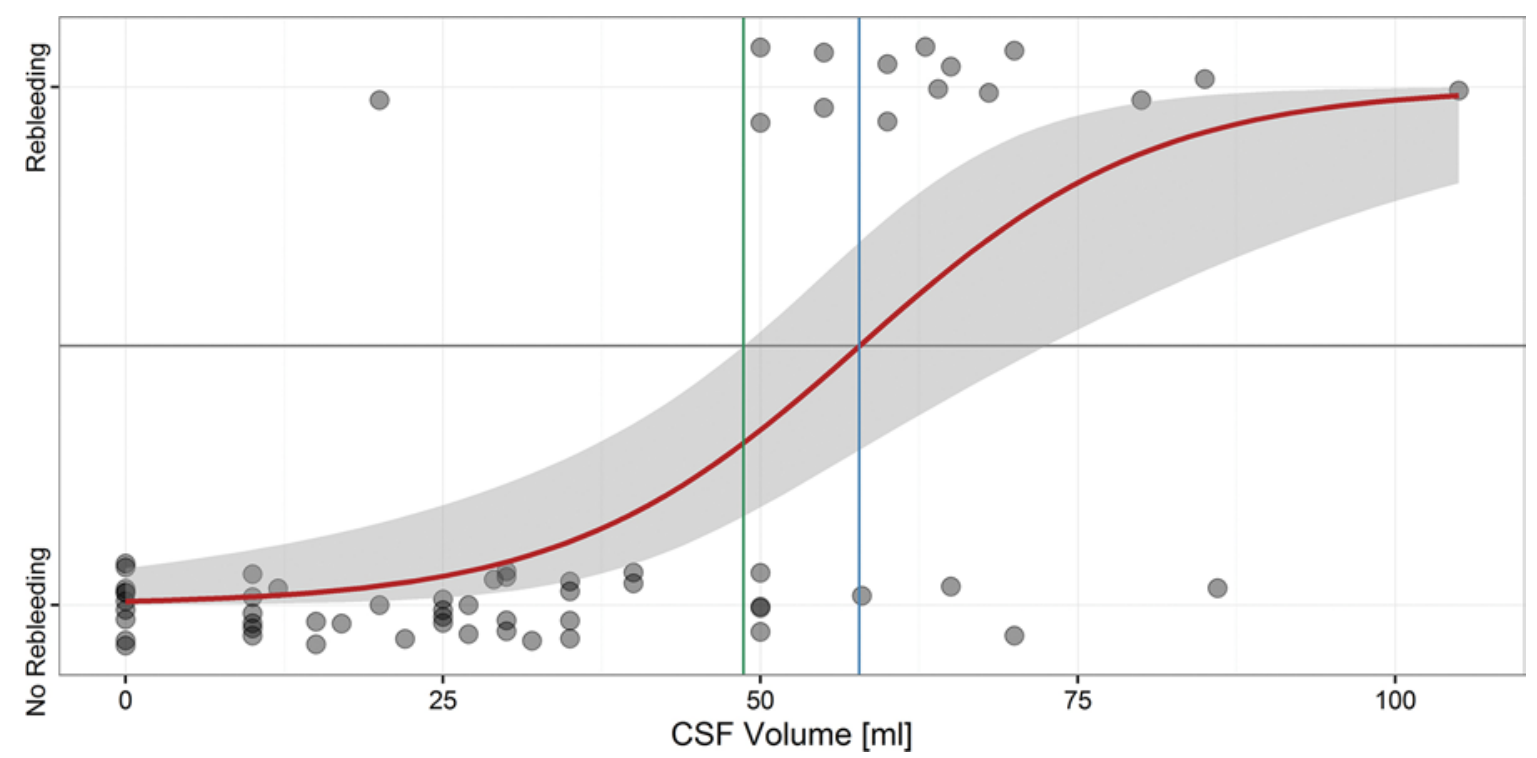

FIG. 4. Graph of the logistic regression for the identification of the rebleeding probability. The right (blue) and left (green) vertical lines represent the cutoff value and the lower range of its $95 \% \mathrm{Cl}$, respectively. Figure is available in color online only. 
However, the use of an EVD remains an undisputed and pertinent tool to treat acute hydrocephalus, and continuous CSF drainage has also been advocated in the acute phase after aSAH to reduce the risk of ischemic complications. ${ }^{11,17}$ Despite this controversy, thresholds for the volume of CSF drainage remain undefined even though there have been multiple reports on the adverse effects of early CSF drainage and accidental overdrainage on the incidence of aneurysmal rebleeding within the first 24 hours, highlighting the need for CSF drainage guidelines with defined thresholds. ${ }^{19,22}$ This volume-mediated effect constitutes one of the most important predictors for rebleeding as demonstrated in our cohort. A volume of drained CSF above $60 \mathrm{ml}$ within the first 6 hours significantly increases the rebleeding risk and should be avoided. In this respect, automated ICP- and volume-controlled CSF drainage systems might reduce the risk of rebleeding in these patients. Interestingly, although insertion of an EVD contributes to the rebleeding risk, timing of EVD placement and duration of monitoring and CSF drainage does not (Table 2).

Both premorbid hypertension and hypertension upon admission are well-known risk factors and are associated with increased severity of the initial bleeding event; these represent significant risk factors for aneurysmal rebleeding. ${ }^{5,20}$ Although hypertension upon and during admission was assessed, we did not include premorbid hypertension in our model. This might have influenced our results because of its significant effect in other reports. In our study, patients with thrombocytopenia are at increased risk of aneurysmal rebleeding. However, this did not improve the validity of risk assessment compared with CSF drainage volume alone. The application of antifibrinolytic drugs prior to EVD placement in patients requiring treatment for acute hydrocephalus should be discussed, as this medication effectively reduces the incidence of rebleeding. ${ }^{1}$ However, the benefits of antifibrinolytic agents are still inconclusive due to a significantly higher rate of delayed cerebral ischemia and the failure of these agents to improve overall survival and functional outcome. ${ }^{1}$ Its effectiveness in this selected situation therefore remains highly speculative. Recent publications show that aneurysm closure within 24 hours after ictus results in a lower rebleeding rate and improves clinical outcome. ${ }^{18}$ However, the median time to aneurysm closure in our population was significantly lower than 24 hours. Aneurysm closure on an emergency basis would be an alternative strategy to reduce rebleeding. ${ }^{16}$

Limitations of this preliminary study are those that are generally associated with the retrospective analysis of small patient cohorts. As a result, we were unable to include patients with short periods of CSF drainage, and exact amounts of CSF volume prior to rebleeding could not be determined. We realize that the cutoff of CSF drainage over a 6-hour period is arbitrary, and it was chosen to allow for uniform analysis between patients. Two patients who re-bled were excluded because of this reason and this might have affected our results. Also, because WFNS grade and aneurysm size were independent predictors for a high volume of CSF drainage, it is possible that these factors were driving the rebleeding events, as these parameters have been linked to aneurysmal rebleeding in previous studies., 32
Moreover, our treatment protocol used an intermittent drainage system and is likely to result in greater drops in ICP. As no continuous ICP values were available, we could not determine whether absolute drops in ICP play a role in the etiology of aneurysmal rebleeding. Finally, it is possible that our study underestimates the incidence of early rebleeding. Although the focus of this study does not lie at preadmission rebleeding, patients in poor clinical condition might have had a rebleeding event without clear clinical signs, but it is unlikely that this would go unnoticed under invasive neuromonitoring.

Despite these limitations, we were able to demonstrate a threshold for CSF drainage as one of the paramount contributors to aneurysmal rebleeding and identify a group of patients in great need of a yet undefined treatment protocol. We believe that the risk for early rebleeding can be affected by an adequate treatment strategy, and thus the protocol for a prospective trial to evaluate the role of controlled automated CSF drainage, the use of antifibrinolytic medication, and ultra-early aneurysm closure is currently under development. The beginning of this study is planned for the near future.

\section{Conclusions}

The most significant factor of in-hospital rebleeding from an unsecured cerebral aneurysm was initiation of CSF drainage and the volume of drained CSF. This necessitates meticulous control of the amount of drained CSF in patients in whom drainage is needed due to acute hydrocephalus. Development of a definitive treatment protocol for these patients is mandatory.

\section{References}

1. Baharoglu MI, Germans MR, Rinkel GJ, Algra A, Vermeulen M, van Gijn J, et al: Antifibrinolytic therapy for aneurysmal subarachnoid haemorrhage. Cochrane Database Syst Rev (8):CD001245, 2013

2. Beck J, Raabe A, Szelenyi A, Berkefeld J, Gerlach R, Setzer $\mathrm{M}$, et al: Sentinel headache and the risk of rebleeding after aneurysmal subarachnoid hemorrhage. Stroke 37:2733-2737, 2006

3. Boogaarts HD, van Lieshout JH, van Amerongen MJ, de Vries J, Verbeek AL, Grotenhuis JA, et al: Aneurysm diameter as a risk factor for pretreatment rebleeding: a metaanalysis. J Neurosurg 122:921-928, 2015

4. Connolly ES Jr, Rabinstein AA, Carhuapoma JR, Derdeyn CP, Dion J, Higashida RT, et al: Guidelines for the management of aneurysmal subarachnoid hemorrhage: a guideline for healthcare professionals from the American Heart Association/American Stroke Association. Stroke 43:1711-1737, 2012

5. De Marchis GM, Lantigua H, Schmidt JM, Lord AS, Velander AJ, Fernandez A, et al: Impact of premorbid hypertension on haemorrhage severity and aneurysm rebleeding risk after subarachnoid haemorrhage. J Neurol Neurosurg Psychiatry 85:56-59, 2014

6. Fujii Y, Takeuchi S, Sasaki O, Minakawa T, Koike T, Tanaka R: Ultra-early rebleeding in spontaneous subarachnoid hemorrhage. J Neurosurg 84:35-42, 1996

7. Guo LM, Zhou HY, Xu JW, Wang Y, Qiu YM, Jiang JY: Risk factors related to aneurysmal rebleeding. World Neurosurg 76:292-298, 253-254, 2011

8. Hasan D, Vermeulen M, Wijdicks EF, Hijdra A, van Gijn J: 
Management problems in acute hydrocephalus after subarachnoid hemorrhage. Stroke 20:747-753, 1989

9. Inagawa $\mathrm{T}$ : Trends in incidence and case fatality rates of aneurysmal subarachnoid hemorrhage in Izumo City, Japan, between 1980-1989 and 1990-1998. Stroke 32:1499-1507, 2001

10. Ingall T, Asplund K, Mähönen M, Bonita R: A multinational comparison of subarachnoid hemorrhage epidemiology in the WHO MONICA stroke study. Stroke 31:1054-1061, 2000

11. Klimo P Jr, Kestle JR, MacDonald JD, Schmidt RH: Marked reduction of cerebral vasospasm with lumbar drainage of cerebrospinal fluid after subarachnoid hemorrhage. J Neurosurg 100:215-224, 2004

12. Larsen CC, Astrup J: Rebleeding after aneurysmal subarachnoid hemorrhage: a literature review. World Neurosurg 79:307-312, 2013

13. Naidech AM, Janjua N, Kreiter KT, Ostapkovich ND, Fitzsimmons BF, Parra A, et al: Predictors and impact of aneurysm rebleeding after subarachnoid hemorrhage. Arch Neurol 62:410-416, 2005

14. Ohkuma H, Tsurutani H, Suzuki S: Incidence and significance of early aneurysmal rebleeding before neurosurgical or neurological management. Stroke 32:1176-1180, 2001

15. Paré L, Delfino R, Leblanc R: The relationship of ventricular drainage to aneurysmal rebleeding. J Neurosurg 76:422427, 1992

16. Park J, Woo H, Kang DH, Kim YS, Kim MY, Shin IH, et al: Formal protocol for emergency treatment of ruptured intracranial aneurysms to reduce in-hospital rebleeding and improve clinical outcomes. J Neurosurg 122:383-391, 2015

17. Park S, Yang N, Seo E: The effectiveness of lumbar cerebrospinal fluid drainage to reduce the cerebral vasospasm after surgical clipping for aneurysmal subarachnoid hemorrhage. J Korean Neurosurg Soc 57:167-173, 2015

18. Phillips TJ, Dowling RJ, Yan B, Laidlaw JD, Mitchell PJ: Does treatment of ruptured intracranial aneurysms within 24 hours improve clinical outcome? Stroke 42:1936-1945, 2011

19. Ruijs AC, Dirven CM, Algra A, Beijer I, Vandertop WP, Rinkel G: The risk of rebleeding after external lumbar drainage in patients with untreated ruptured cerebral aneurysms. Acta Neurochir (Wien) 147:1157-1162, 2005
20. Solanki C, Pandey P, Rao KV: Predictors of aneurysmal rebleed before definitive surgical or endovascular management. Acta Neurochir (Wien) 158:1037-1044, 2016

21. Steiner T, Juvela S, Unterberg A, Jung C, Forsting M, Rinkel G: European Stroke Organization guidelines for the management of intracranial aneurysms and subarachnoid haemorrhage. Cerebrovasc Dis 35:93-112, 2013

22. van Donkelaar CE, Bakker NA, Veeger NJ, Uyttenboogaart M, Metzemaekers JD, Luijckx GJ, et al: Predictive factors for rebleeding after aneurysmal subarachnoid hemorrhage: rebleeding aneurysmal subarachnoid hemorrhage study. Stroke 46:2100-2106, 2015

23. Voldby B, Enevoldsen EM: Intracranial pressure changes following aneurysm rupture. Part 3: Recurrent hemorrhage. J Neurosurg 56:784-789, 1982

\section{Disclosures}

The authors report no conflict of interest concerning the materials or methods used in this study or the findings specified in this paper.

\section{Author Contributions}

Conception and design: van Lieshout, Pumplün, Petridis. Acquisition of data: van Lieshout, Pumplün, Beseoglu. Analysis and interpretation of data: van Lieshout, Fischer, Beseoglu. Drafting the article: van Lieshout, Boogaarts, Beseoglu. Critically revising the article: van Lieshout, Fischer, Kamp, Cornelius, Steiger, Boogaarts, Petridis, Beseoglu. Reviewed submitted version of manuscript: all authors. Approved the final version of the manuscript on behalf of all authors: van Lieshout. Statistical analysis: van Lieshout, Fischer, Beseoglu. Administrative/technical/material support: van Lieshout, Steiger. Study supervision: van Lieshout, Petridis, Beseoglu.

\section{Correspondence}

Jasper Hans van Lieshout, Department of Neurosurgery, HeinrichHeine University Düsseldorf, Moorenstrasse 5, Düsseldorf 40225, Germany. email: jasper.vanlieshout@med.uni-duesseldorf.de. 